\title{
REPEAT-PASS SPACEBORNE TRANSMITTER-STATIONARY RECEIVER BISTATIC SAR INTERFEROMETRY - FIRST RESULTS
}

\author{
Andrei Anghel ${ }^{\star} \quad$ Remus Cacoveanu ${ }^{\star} \quad$ Mihai Datcu $^{\star \ddagger}$ \\ * University POLITEHNICA of Bucharest, 1-3 Iuliu Maniu, Bucharest, Romania \\ $\ddagger$ German Aerospace Center (DLR), Oberpfaffenhofen, Germany
}

\begin{abstract}
This paper presents the first results obtained by repeat-pass bistatic synthetic aperture radar (SAR) interferometry using a fixed C-band ground-based receiver and the Sentinel-1A/B satellites as transmitters of opportunity. The methodology developed to obtain repeat-pass bistatic SAR interferograms uses as input a stack of range compressed bistatic acquisition data and mainly consists in the following stages: raw interferograms computation on a two-dimensional grid in ground geometry, atmospheric phase screen removal and topographic phase compensation. The displacements of a high-rise building were estimated using two stacks of bistatic SAR images acquired between April-June 2017 over an area of Bucharest city, Romania.
\end{abstract}

Index Terms - Repeat-pass, Bistatic SAR, Interferometry.

\section{INTRODUCTION}

Bistatic synthetic aperture radar (SAR) imaging systems with a stationary receiver and a spaceborne transmitter have been recently studied in literature. Among the satellites used as transmitters of opportunity for bistatic SAR imaging, the most common are: TerraSAR-X [1, 2], Sentinel-1A/B [3, 4], GNSS [5] and ERS-2/ENVISAT [6]. Most of the works concerning this type of bistatic SAR are focused on image formation procedures and single-pass interferometry/tomography. A first study of repeat-pass bistatic SAR interferometry was performed in [6] where coregistration and temporal decorrelation are discussed. An analysis of the repeat-pass bistatic interferometric phase sensitivity to height and displacement was presented in [7], among with the first raw bistatic interferograms obtained with Sentinel-1A/B.

One of the assets of bistatic geometry is the ability to use as transmitters of opportunity different satellites flying on various orbits, which leads to new perspectives concerning target characterization and tracking relative to monostatic geometry (i.e., a target that is not visible in a monostatic image may be visible in a bistatic image if the ground receiver is properly positioned, and the revisit time can be enhanced if multiple orbits are exploited).
This paper proposes a methodology to obtain repeatpass bistatic SAR interferograms using as inputs range compressed images obtained with the platform presented in [4]. The method involves the following steps: raw interferograms computation on a two-dimensional grid in ground geometry, atmospheric phase screen removal and topographic phase compensation. The approach is applied on two stacks of images acquired on two different parallel orbits of the Sentinel1A/B satellites between April-June 2017 over an area of Bucharest city, Romania. The displacement time series of a high-rise building is extracted from the interferograms.

The remainder of the paper is structured as follows. Section II presents the developed procedure to generate repeatpass bistatic SAR interferograms, whereas in Section III the methodology is applied to real data. The conclusions are stated in Section IV.

\section{REPEAT-PASS BISTATIC INTERFEROGRAMS GENERATION}

The geometry considered for repeat-pass bistatic SAR inteferometry is shown in Fig. 1. The ground receiver has two channels -one that receives directly the transmitted pulses through an antenna pointing towards the satellite (reference channel) and another that receives the reflected signals through an antenna oriented towards the illuminated scene (usually called imaging channel). The depicted baselines are computed in the same way as in monostatic interferometry. The high level

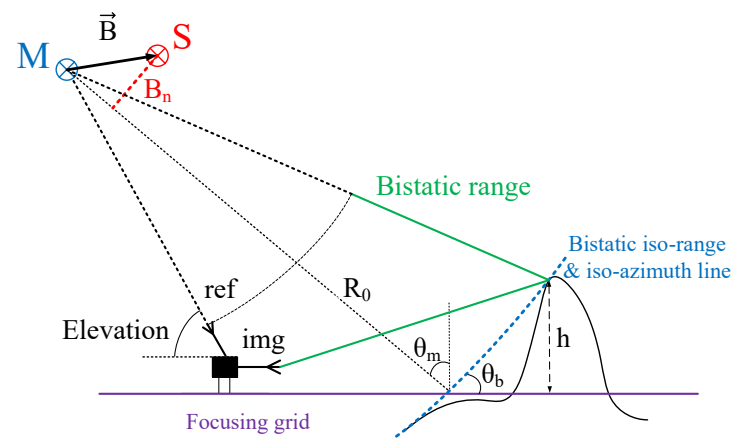

Fig. 1. Envisaged geometry for repeat-pass bistatic SAR interferometry 
block diagram of the procedure used to generate repeat-pass bistatic interferograms is shown in Fig. 2, and all the steps are described according to the flow on the processing chain.

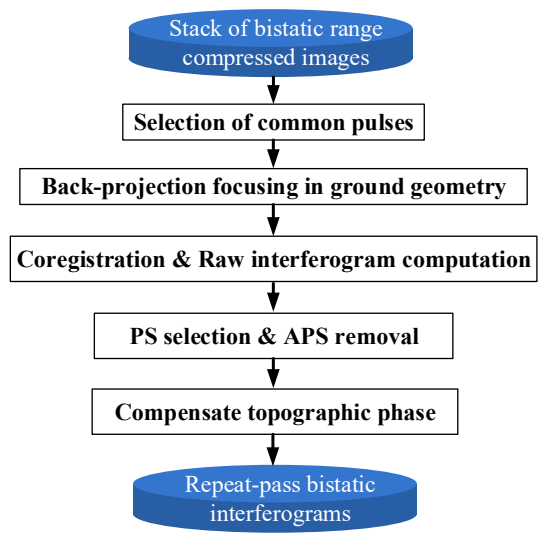

Fig. 2. Methodology used to generate repeat-pass bistatic SAR interferograms

The stack of synchronized range compressed data required as input for the interferograms generation procedure is obtained using the algorithm described in [4]. Additionally, for each pulse received by the ground platform (corresponding to a row in a range image matrix) we know an estimate of the satellite's position vector at the moment when the respective pulse was transmitted. Using this information, for each acquisition only the pulses that are common to all other acquisitions in the stack are selected to be used in the backprojection focusing algorithm, which acts like a common azimuth bandwidth filtering (leading to a mismatch of at most 1 pulse). The azimuth focusing is performed on a uniform twodimensional cartesian grid given in Earth-Centered-EarthFixed (ECEF) coordinates. The focused bistatic SAR images are coregistered by means of inter-correlation performed on the area of interest.

The permanent scatterers (PS) selection in the stack of coregistered bistatic images is performed by testing the temporal amplitude stability of each pixel (the classical manner used in SAR interferometry [8]). The amplitude dispersion index $\left(D_{A}\right)$ of every pixel is computed for the given stack, and the pixels selected as PSs are the ones for which $D_{A} \leq D_{t h}$, where $D_{t h}$ is a given threshold (typically chosen 0.25 ). If the SAR image is focused on a relatively dense grid compared to the point spread function's width, a target's lobe may cover several pixels. Hence, the dispersion index is actually computed only for the regional maxima of the master amplitude image.

The raw interferograms are obtained from the complex coherence computed between each image and the first image of the stack (considered master image). To estimate the atmospheric phase screen (APS) of each raw interferogram we consider that the atmosphere is spatially homogeneous between the ground receiver and the illuminated scene. This hypothesis is usually employed for ground-based interferometric radars $[9,10]$, especially in urban scenarios. It can be shown that for distances placed at a few kilometers from the ground receiver, this model can also be applied as a good approximation for spaceborne transmitter-ground receiver bistatic geometry, and the target phase can be written as [11]:

$$
\varphi(t)=\varphi_{0}+\frac{2 \pi}{\lambda} \int_{L_{p}} n(t) d l=\varphi_{0}+\frac{2 \pi}{\lambda} n(t) R_{b},
$$

where $\varphi_{0}$ is the target's backscattering phase, $\lambda$ is the wavelength at central frequency, $L_{p}$ is the propagation path, $n(t)$ is the refractive index (assumed to be only time-dependent) and $R_{b}$ is the bistatic range (the sum between the satellite-target and target-receiver distances minus the direct satellite-ground receiver path). Hence the atmospheric phase screen that we need to estimate will be a linear function of the bistatic range $R_{b}$ depending on the refractive index variation $n\left(t_{2}\right)-n\left(t_{1}\right)$ between two acquisitions made at the moments $t_{1}$ and $t_{2}$. In the following, we consider that the interferometric phase screen has the form:

$$
\Delta \varphi\left(t_{1}, t_{2}, R_{b}\right)=a_{0}+a_{1} R_{b} .
$$

The APS estimation algorithm has to determine two parameters -the phase slope $a_{1}$ and the phase offset $a_{0}$ (which can also include offsets determined by geometric errors or group delay variations with temperature of the RF cables between the ground-based receive antennas and the digitizer). In works concerning ground-based interferometry, a linear regression is applied to determine the two parameters (e.g., in [9]). However, if the targets are not very close to the radar or the phase slope is relatively high, several phase wraps can occur and the linear model is valid only on subintervals that are not apriori known. Hence, we propose to estimate the APS using a cost function defined as:

$$
J\left(a_{0}, a_{1}\right)=\sum_{i=1}^{N_{P S}}\left|\frac{A_{i}}{\left|A_{i}\right|}-\exp \left[j\left(a_{0}+a_{1} R_{b, i}\right)\right]\right|^{2},
$$

where $N_{P S}$ is the number of identified PSs, $A_{i}$ and $R_{b, i}$ are the complex amplitude and, respectively, the bistatic range of PS $i$. This function considers the phase of each PS relative to the linear phase model, both being expressed as complex exponentials, which avoids phase wraps. Note that this function is similar to the error vector magnitudes (EVM) used in digital communications to quantify the performance of a transmitter/receiver. The estimated APS parameters for each interferometric pair are the arguments that minimize the cost function.

To obtain the final interferograms, the APS is removed and the topographic phase is compensated using the phase sensitivity to height computed in [7]:

$$
\frac{\Delta \varphi}{\Delta h}=\frac{2 \pi}{\lambda} \frac{B_{n}}{R_{0}} \frac{\cos \left(\theta_{b}-\theta_{m}\right)}{\sin \left(\theta_{b}\right)}
$$


in which $B_{n}$ is the normal baseline, $R_{0}$ is the closest approach distance for the master image, $\theta_{m}$ is the incidence angle and $\theta_{b}$ is the equivalent bistatic incidence angle (the angle between the ground plane and the iso-range/iso-azimuth line, as shown in Fig. 1). Finally, the interferometric phases for the considered targets are converted to bistatic distances (the sum of the projections of the real distance on the two line of sights: satellite-target and ground receiver-target).

\section{EXPERIMENTAL RESULTS}

This section presents the repeat-pass bistatic SAR interferometric results obtained for two stacks of images acquired over an area of Bucharest city, Romania, between April-June 2017 with the platform presented in [4] and the Sentinel-1A/B satellites. The ground receiver was mounted on the roof of the Politehnica university rectorate building at an altitude of around $100 \mathrm{~m}$.

In Sentinel's TOPSAR operating mode, a region on the ground is usually visible from one sub-swath of one orbit. However, in the bistatic configuration the same region can be illuminated from more orbits, and for each orbit from several sub-swaths (the ground receiver can capture pulses even if the satellite's main lobe is not pointed towards it). In the following we describe each orbit by its elevation angle (from the ground receiver position) and each sub-swath by its central azimuth time $t_{a z}$ relative to the closest approach position. The main ascending orbit (for which the satellite beam is pointed towards the ground receiver) is observed at an elevation of $52^{\circ}$, whereas a secondary ascending orbit is visible at an elevation of $43^{\circ}$ (for this orbit, the region surrounding the ground receiver does not appear at all in the monostatic image). The stack acquired for the orbit with $52^{\circ}$ elevation has 15 images, while the one for $43^{\circ}$ elevation contains 13 images. An example of the pulses captured for the two orbits are shown in Fig. 3(a) and Fig. 3(b). The upper plots of each figure show the nominal pulse repetition interval (PRI) of each sub-swath and the PRI computed as difference between consecutive GPS timestamps stored by the ground receiver, whereas the lower plots display the amplitude of each received pulse. Note that the closest approach point (zero azimuth time) appears in both cases for sub-swath 2 (with 689us PRI), but we also receive pulses in sub-swaths 1 and 3 . The azimuth focusing and further processing is performed separately for each selected group of consecutive pulses (e.g., the groups of pulses around the closest approach time are highlighted in green in the lower plots of Fig. 3(a) and Fig. 3(b)). Four sets of interferograms were generated in total (two for each orbit): for the $43^{\circ}$ elevation orbit we considered the groups of pulses centered on $t_{a z}=0.07 \mathrm{~s}$ and $t_{a z}=0.83 \mathrm{~s}$, while for the $52^{\circ}$ case we considered the groups centered on $t_{a z}=-0.05 s$ and $t_{a z}=-0.80 s$.

The focusing grid is aligned with the local latitude and longitude, has a $2 \mathrm{~m} \times 2 \mathrm{~m}$ resolution and the same altitude

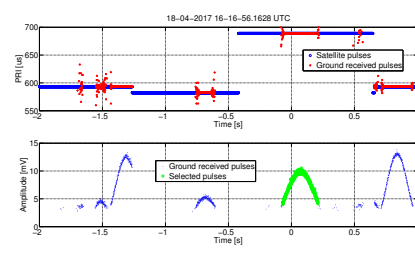

(a)

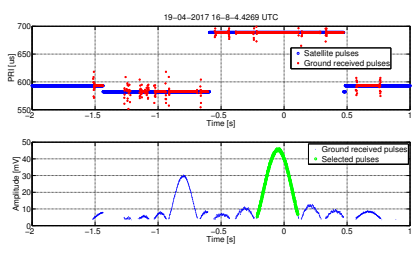

(b)
Fig. 3. The PRI (nominal and computed from the timestamps) and the amplitude for the acquisitions from (a) 18.04.2017 (43 ${ }^{\circ}$ elevation) and (b) 19.04.2017 (52 ${ }^{\circ}$ elevation).

as the receiver. The position of the receiver is taken as origin for the latitude/longitude axis. Fig. 4 presents an amplitude image of the illuminated area (among with the selected PSs). The near-grazing receive geometry in the imaged scene favors reflections from targets that rise above the horizontal plane situated at the ground receiver's altitude.

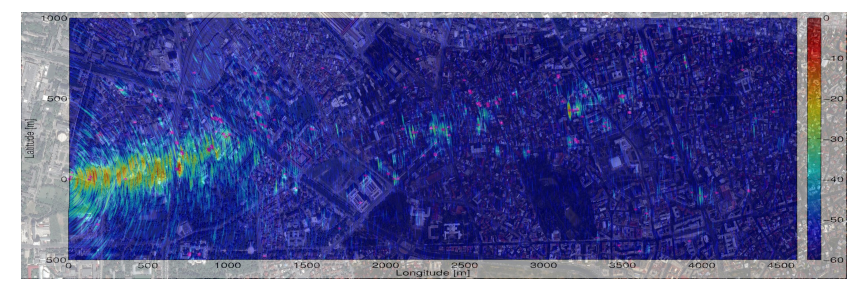

Fig. 4. Amplitude image acquired on 01.04.2017 among with the selected PSs for a dispersion index of 0.25 .

The displacement time series was extracted from the interferograms for a highly reflective target with a height of $75 \mathrm{~m}$, the Cathedral plaza building. The response of this building in the 4 cases considered is shown in Fig. 5. Notice that the shape of the response depends on the orbit and the sub-swath, which underlines the diversity that can be exploited in bistatic geometry (moreover, the building is not distinguishable in the monostatic images acquired from the orbit with $52^{\circ}$ elevation). The interferometric phase is extracted from the highest peak of the west side. Fig. 6 displays the estimated displacements of the Cathedral plaza building among with the temperatures at the acquisition dates. The curves corresponding to the same orbit and different sub-swath are highly correlated, while two curves from different orbits are a bit less correlated, but the general trend is the same. This aspect can be linked to at least two facts: the acquisitions on one orbit are one day shifted from the acquisitions on the other, and the phase centers may not be the same for the two orbits. All the curves show an average evolution in keeping with the temperature variation, which can be explained by the increase of the building's height due to dilatation. The mentioned correlations can be viewed as a qualitative validation of the proposed repeatpass bistatic interferograms generation procedure. 


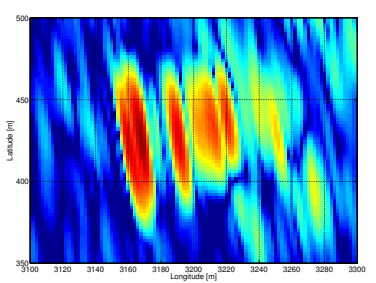

(a)

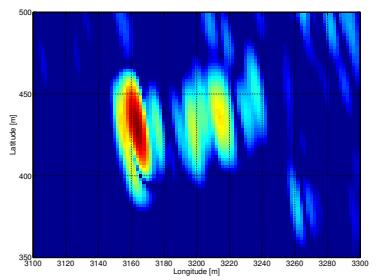

(c)

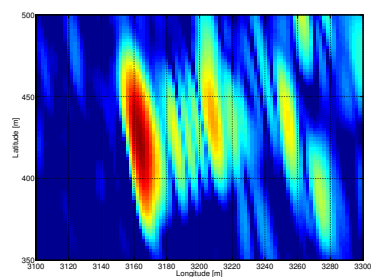

(b)

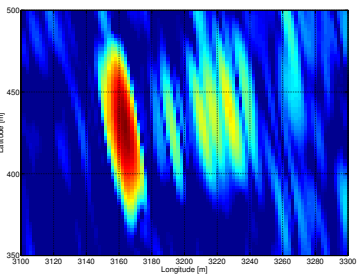

(d)
Fig. 5. Cathedral plaza building imaged in 4 cases: (a) $43^{\circ}$ elevation, $t_{a z}=0.07 \mathrm{~s}$, (b) $43^{\circ}$ elevation, $t_{a z}=0.83 \mathrm{~s}$, (c) $52^{\circ}$ elevation, $t_{a z}=-0.05 \mathrm{~s}$, (d) $52^{\circ}$ elevation, $t_{a z}=-0.80 \mathrm{~s}$.

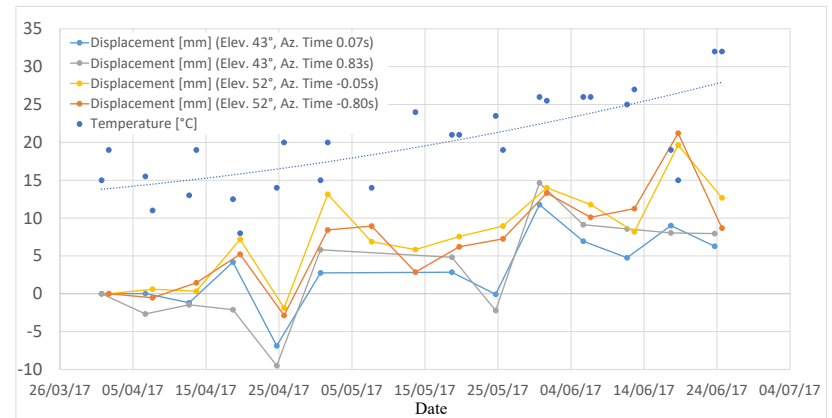

Fig. 6. Displacement time series [mm] for the Cathedral plaza building between April-June 2017 and the temperatures $\left[{ }^{\circ} \mathrm{C}\right]$ at the dates of acquisition.

\section{CONCLUSIONS}

This paper presented a methodology to obtain repeat-pass bistatic SAR interferograms and the first experimental results obtained with two stacks of images acquired from two parallel orbits of the Sentinel-1A/B satellites. The possibility to exploit multiple orbits and multiple sub-swath from the same ground location in repeat-pass bistatic SAR interferometry was demonstrated. An in-situ validation campaign of the system's measurement capabilities is currently in progress. Future work will focus on combining the signals from different orbits/sub-swaths to enhance various imaging parameters.

\section{ACKNOWLEDGMENT}

This work has been funded by the European Space Agency through the COBIS project, Ctr. No. 4000115608/15/NL/CBi and by University Politehnica of Bucharest, through the Excellence Research Grants Program, UPB GEX 2017, Identifier: UPB- GEX2017, Ctr. No. 37/25.09.2017 (AMIRAD project).

\section{REFERENCES}

[1] F. Behner, S. Reuter, H. Nies, and O. Loffeld, "Synchronization and Processing in the HITCHHIKER Bistatic SAR Experiment," IEEE J. Sel. Topics Appl. Earth Observ. Remote Sens., vol. 9, no. 3, pp. 1028-1035, 2016.

[2] A. Anghel et al., "Simplified bistatic SAR imaging with a fixed receiver and TerraSAR-X as transmitter of opportunity - first results," in IEEE IGARSS, Beijing, China, 2016, pp. 2094-2097.

[3] V. Kubica, X. Neyt, and H. Griffiths, "Improved crossrange resolution in TOPSAR imaging using Sentinel-1A in bistatic operation," in IEEE Radar Conference, Arlington, VA, USA, 2015, pp. 0117-0122.

[4] A. Anghel et al., "Bistatic SAR imaging with Sentinel-1 operating in TOPSAR mode," in IEEE Radar Conference, Seattle, WA, USA, May 2017, pp. 601-605.

[5] X. Fan et al., "Passive SAR with GNSS transmitters: Latest results and research progress," in IEEE IGARSS, July 2017, pp. 1043-1046.

[6] S. Duque et al., "Repeat-pass interferometry using a fixed-receiver and ERS-2/ENVISAT as transmitters of opportunity," in IEEE IGARSS, Cape Town, South Africa, July 2009, vol. 2, pp. II-246-II-249.

[7] A. Anghel et al., "Phase sensitivity analysis of spaceborne transmitter - stationary ground-based receiver bistatic SAR interferometry with one imaging channel," in IEEE IGARSS, July 2017, pp. 1051-1054.

[8] A. Ferretti, C. Prati, and F. Rocca, "Permanent scatterers in SAR interferometry," IEEE Trans. Geosci. Remote Sens., vol. 39, no. 1, pp. 8-20, Jan 2001.

[9] L. Pipia et al., "Atmospheric Artifact Compensation in Ground-Based DInSAR Applications," IEEE Geosci. Remote Sens. Lett., vol. 5, no. 1, pp. 88-92, Jan 2008.

[10] L. Noferini et al., "Permanent scatterers analysis for atmospheric correction in ground-based SAR interferometry," IEEE Trans. Geosci. Remote Sens., vol. 43, no. 7, pp. 1459-1471, July 2005.

[11] L. Iannini and A. Monti Guarnieri, "Atmospheric Phase Screen in Ground-Based Radar: Statistics and Compensation," IEEE Geosci. Remote Sens. Lett., vol. 8, no. 3, pp. 537-541, May 2011. 